\title{
AUTOESTIMA E ASPECTOS SOCIOEMOCIONAIS EM PACIENTES DE CIRURGIA ORTOGNÁTICA: PROPOSTAS DE ANÁLISE PSICOLÓGICA
}

\section{Matheus Sousa de Macena}

Graduado em Psicologia pela Universidade Tiradentes (SE). Mestrando em Processos de Desenvolvimento Humano e Saúde na Universidade de Brasília, Brasília (DF), Brasil.

\section{Letícia Dultra de Faro Menezes}

Aluna de iniciação científica. Acadêmica do curso de Psicologia da Universidade Tiradentes, Aracaju (SE), Brasil.

\section{Marcelo Rodrigo de Souza Melo}

Graduado em Odontologia pela Universidade Federal de Sergipe e Especialista em Cirurgia e Traumatologia Buco-Maxilo-Facial pelo Colégio Brasileiro de Cirurgia e Traumatologia Buco-Maxilo-Facial, São Paulo (SP), Brasil.

\section{Lívia de Melo Barros}

Doutora em Educação pela Pontifícia Universidade Católica do Rio Grande do Sul (RS). Docente no curso de Psicologia da Universidade Tiradentes, Aracaju (SE), Brasil.
RESUMO: A pesquisa objetivou investigar a autoestima e os aspectos socioemocionais de pacientes com deformidades dentofaciais submetidos à cirurgia ortognática. Disponibilizamos um questionário da plataforma Google Docs em um grupo de uma rede social composto por pessoas com interesses em comum no tema. Houve 120 respostas no total e, em geral, elas revelaram que houve aumento de autoestima nos pacientes (média geral subiu de 4,58 para 8,33) e melhora significativa em fatores funcionais, sociais e psicológicos. Contudo, existe a possibilidade de frustração para alguns sujeitos submetidos ao procedimento, oriundo das expectativas perante a cirurgia, especialmente no âmbito estético. A cirurgia ortognática é uma alternativa considerável no tratamento de deformidades dentofaciais e de seus agravos, porém, diante da pluralidade de resultados e reações, infere-se a importância de acompanhamento psicológico no pré e póscirúrgico, como forma de preparo para a cirurgia e de adaptação aos resultados do procedimento.

PALAVRAS-CHAVE: Cirurgia ortognática; Impacto psicossocial; Autoestima.

\section{SELF-ESTEEM AND SOCIO-EMOTIONAL ASPECTS IN ORTHOGNACTIC SURGERY PATIENTS: PROPOSALS FOR PSYCHOLOGICAL ANALYSIS}

\begin{abstract}
This study aimed to investigate the self-esteem and the socio-emotional aspects of patients with dentofacial deformities submitted to orthognathic surgery. We provide a Google Docs platform questionnaire in a social network group composed of people with common interests. There were 120 responses in total and, in general, there was an increase in self-esteem in the patients (general average increased from 4.58 to 8.33 ) and significant improvement in functional, social and psychological factors. There is, however, the possibility of that some subjects submitted to the procedure may experience frustration, arising from expectations before the surgery, especially aesthetic ones. Orthognathic surgery is a viable alternative in the treatment of dentofacial deformities and their aggravations. However, given the plurality of results and reactions, we infer the importance of psychological follow-up in preoperative and postoperative periods as preparation for surgery and adaptation to the results of the procedure.
\end{abstract}

KEY WORDS: Orthognatic surgery; Psychosocial impact; Self-esteem. 


\section{INTRODUÇÃO}

A cirurgia ortognática é um procedimento de tratamento para deformidades dentofaciais, melhora da oclusão, adequação do posicionamento dos maxilares e harmonização facial, além de poder proporcionar benefícios à condição respiratória, elevação da autoestima e, consequentemente, maior satisfação estética e funcional ${ }^{1}$. Os resultados de um estudo sobre esse tema demonstraram que a cirurgia ortognática influencia de maneira muito positiva o humor do indivíduo. Especificamente, quando os pacientes se posicionaram sobre sua aparência física após a cirurgia, apresentaram um nível maior de felicidade e demonstraram alto senso de controle e de poder sobre suas vidas ${ }^{2}$.

Uma das extensões psicológicas que têm mostrado relevância na odontologia e, particularmente, nos indivíduos com deformidade dentofacial sujeitos à cirurgia ortognática é a autoestima. Sendo assim, o cirurgião-dentista deve enxergar os seus pacientes como indivíduos biopsicossociais ${ }^{3}$. A autoestima é um fator determinante para o modo como as pessoas valorizam a si mesmas ou o outro e suas relações, e também para as suas reações ao ambiente em inúmeros contextos. Diante disso, é notória sua influência na qualidade de vida, bemestar psicológico e saúde mental dos indivíduos em geral ${ }^{4}$.

A autoestima pode ainda ser descrita como o sentimento, o apreço e a consideração que a pessoa sente por si própria, como ela se percebe e o que pensa sobre si mesma 5 . Já os aspectos socioemocionais estão associados à capacidade de a pessoa relacionar-se consigo mesma e com os outros, sendo responsável em seus comportamentos e atitudes, mantendo autocontrole emocional e resiliência. Isso posto, vê-se que tais aspectos impactam diversos âmbitos da vida do sujeito ${ }^{6-7}$.

Dessa forma, a construção dos aspectos socioemocionais de pacientes submetidos à cirurgia ortognática pode evidenciar a necessidade de atendimento multiprofissional em todas as etapas do tratamento, partindo do pré-operatório e prosseguindo no pós-operatório em cirurgia ortognática. Uma vez que o paciente não será modificado apenas fisicamente, os fatores socioemocionais transcendem a intervenção cirúrgica e podem ocasionar tanto consequências benéficas quanto maléficas aos pacientes.
Os objetivos deste trabalho foram investigar a autoestima e os aspectos socioemocionais de pacientes com deformidades dentofaciais submetidos à cirurgia ortognática; identificar as mudanças de comportamento do paciente; analisar alterações emocionais após o procedimento cirúrgico; verificar quais os impactos na autoestima do paciente; investigar fatores determinantes do trabalho do profissional de psicologia com pacientes de cirurgia ortognática.

Ainda é restrita a produção de materiais sobre psicologia aplicada à odontologia. Apesar de ser comum encontrar na literatura temas abrangentes sobre as duas áreas do conhecimento, temas específicos que relacionam os impactos psicológicos em pacientes submetidos à cirurgia ortognática ainda possuem um déficit de estudos nacionais e internacionais. Desse modo, é entendido que há uma carência em ambas as áreas sobre o tema.

\section{METODOLOGIA}

É um estudo do tipo descritivo com delineamento transversal e tratamento realizado por meio da análise textual discursiva. Os dados foram coletados mediante aplicação de um questionário semiestruturado disponibilizado em uma plataforma online a pacientes que foram submetidos à cirurgia ortognática. As respostas atribuídas pelos participantes foram categorizadas de acordo com os indicadores psicológicos que emergiram nos pacientes com deformidades dentofaciais antes e após a intervenção cirúrgica.

O instrumento foi construído na plataforma Google Docs e disponibilizado em uma rede social que possui um grupo virtual composto por pessoas que têm o interesse em comum de discutir sobre a cirurgia ortognática, fazendo parte desse grupo indivíduos que vivem em todo o território nacional. Foram obtidas respostas de 120 participantes, no período entre outubro/2016 e novembro/2018.

Perguntou-se sobre a vida antes e depois da cirurgia, incluindo indicadores emocionais e funcionais e relações interpessoais. Os participantes puderam responder sobre quais fatores os levaram a serem submetidos à cirurgia ortognática; sobre as emoções no pré e no pós-cirúrgico; avaliar quantitativamente a 
autoestima nesse mesmo período; descrever a própria vida antes e após a cirurgia; e relatar se houve resistência em se sujeitar ao procedimento, o apoio recebido da família e se teve acompanhamento psicológico em algum momento. Foram indicados nas respostas os aspectos mais afetados na vida dos participantes. As perguntas quantitativas seguiram para uma média aritmética dos resultados.

Utilizou-se como critério de inclusão para a análise dos questionários a submissão à cirurgia ortognática e a idade acima de 16 anos. $O$ critério de exclusão foi a não submissão ao procedimento cirúrgico.

Para a análise dos dados, empregou-se a análise textual discursiva de Moraes e Galiazzi ${ }^{8}$. É importante ressaltar que, ao optar-se por uma metodologia que também possui um caráter qualitativo, utilizando o método da análise textual discursiva, assume-se uma perspectiva fenomenológica, segundo a qual os fenômenos se manifestam de forma livre, sem que sejam impostos direcionamentos, o que torna a leitura dos dados verossímil e dialética ${ }^{8}$. Os dados foram divididos em quatro categorias: autoestima; comportamento social; emoções associadas; fatores estéticos.

O passo seguinte foi o processo de imersão nos resultados, sendo que, nessa etapa, ocorre a unitarização e a impregnação dos dados. Em tais procedimentos, foi necessário desorganizar a estrutura do corpus, a fim de que houvesse uma leitura e uma análise dos dados aprofundada. Essa ação permitiu uma nova associação entre os elementos unitários e a produção de uma nova ordem, significando novos entendimentos sobre o fenômeno estudado.

Após a categorização dos resultados, ocorreu a ascensão do novo, que é um processo auto-organizado e intuitivo que envolve compreensões, interpretações e análise do material implícito. Esse tipo de análise, a textual discursiva, possibilitou trabalhar com os significados explícitos pelos pesquisados, como também proporcionou uma análise da demanda latente ou implícita.

A fase subsequente consistiu em comunicar as compreensões emergentes. Os pesquisadores produziram novos significados e sentidos que são expostos por meio da linguagem escrita de modo complexo. É válido pontuar que o recorte e a percepção dos pesquisadores podem gerar um novo entendimento sobre os sentidos expressos pelos participantes. O objetivo dessa etapa é que seja construído um metatexto, matriciado no corpus inicial que foi reformulado, fragmentado e relacionado com outros discursos, além de que se dê a ampliação das pontuações dos pesquisadores, com base na literatura acerca do fenômeno estudado.

A presente pesquisa foi aprovada pelo Comitê de Ética em Pesquisa sob o parecer $n^{0} 1.723 .722$ e foi conduzida de acordo com os padrões éticos exigidos.

\section{RESULTADOS}

Os resultados da pesquisa foram obtidos a partir das respostas de 120 participantes que responderam ao questionário na plataforma Google Docs. Pelos relatos dos pacientes, foi possível avaliar aspectos relevantes referentes ao pré e pós-cirúrgico no ponto de vista da psicologia e, desse modo, propor práticas de cuidado e tratamento numa ótica de saúde psíquica, por meio do conceito de autoestima.

\section{AUTOESTIMA}

Em uma escala de 0 a 10 , a nota dada para a autoestima, no período pré-cirúrgico, foi 4,58. Já no póscirúrgico, foi 8,33, confirmando-se o impacto psicológico positivo que a cirurgia ortognática pode proporcionar ao paciente. No entanto, 14 sujeitos (11.6\%) indicaram nota abaixo de 7,0, apontando a insatisfação com relação ao resultado e o impacto do procedimento na qualidade de vida destes. Dentre as principais causas dos referidos índices, os sujeitos relataram dificuldades do pós-cirúrgico (por exemplo, inchaço e parestesia,) e, em especial, um resultado estético diferente do esperado.

\section{COMPORTAMENTO SOCIAL}

Ao analisarem-se os dados dos sujeitos que relataram vergonha ao sorrir e/ou falar, foi verificado que, similarmente, eles também não tiram muitas fotos, têm dificuldade de sorrir, tiram fotos de longe ou tentam disfarçar. Dessa maneira, diversos participantes da 
pesquisa demonstraram utilizar de artifícios para tentar amenizar o incômodo estético. Contudo, isso afeta, também, os comportamentos e as relações interpessoais, assim como a qualidade de vida do indivíduo.

Os sujeitos, após a cirurgia, em sua maioria, obtiveram avanço no convívio social, como se pode observar a partir dos seguintes relatos: "Período de [...] novas descobertas e possibilidades de desenvolvimento pessoal, profissional e social, além da alegria em conquistar melhor condição de vida"; "O sentimento de timidez se fazia presente na minha vida pessoal, profissional e social". O primeiro relato aponta a visão de que o paciente adquiriu otimismo em relação ao futuro e às expectativas positivas, enquanto o segundo enfatiza o comportamento perante as pessoas antes da cirurgia e que a insatisfação estética prejudicava o indivíduo em diversas esferas de sua vida.

No entanto, pode-se enfatizar as diferenças entre pacientes que foram operados recentemente e aqueles que já possuem maior tempo de cirurgia. Sobre os recém-operados, estes relatam ainda estarem se adaptando à nova estrutura esquelética facial e à nova aparência. Ressaltam também o inchaço do pósoperatório e, consequentemente, a incidência de dores em geral. Uma minoria de participantes pontuou que sofrem parestesia e/ou insensibilidade no lábio inferior pelas lesões nervosas: "Hoje me sinto muito feliz com o resultado, apesar de ainda ter que lidar com um pouco de parestesia e algumas sensações estranhas".

\section{EMOÇÕES ASSOCIADAS}

De acordo com os dados obtidos pelo questionário, foi constatado que houve diversidade em relação aos tipos de emoções apontadas, bem como uma grande oscilação de emoções a depender do período referente à cirurgia, seja no pré ou no pós-operatório. No tocante à etapa do pré-operatório, foi possível verificar maior variação nas frequências de respostas voltadas às emoções, sendo as principais e, por conseguinte, as mais apontadas no pós-cirúrgico a alegria, o medo, a insegurança, o alívio, a satisfação e a angústia. Acerca do pós-operatório, foi visto que esta é a etapa na qual ocorre menor oscilação e que os números estão mais próximos um do outro. Ainda foi apontado um crescimento notável de angústia, tristeza, timidez, alívio, frustração, surpresa e espanto nessa etapa, e, quando foi perguntado acerca das emoções sentidas meses após a cirurgia, houve maior frequência de respostas relacionadas ao bemestar e à satisfação. Ademais, grande parte dos sujeitos demonstraram elevados índices de expectativa e de incerteza com relação aos resultados esperados.

A satisfação manifestou crescimento ( 57 respostas para "dias antes da cirurgia", 70 para "pós-operatório" e 83 para "alguns meses após a cirurgia") com o decorrer das etapas destacadas no estudo. Em primeira instância, a frequência de resposta relativa a esse quadro emocional no período antes da cirurgia aponta um nível menor de satisfação dos sujeitos, muitas vezes em decorrência do incômodo estético e dos problemas fisiológicos e anatômicos, bem como dos aspectos depressivos, de baixa autoestima e mal-estar, os quais são sentimentos desencadeados pela emoção primária "tristeza". Após a cirurgia, um dos participantes declarou que "as questões funcionais foram surpreendentes, mastigação perfeita, respiração satisfatória, digestão normal".

\section{FATORES ESTÉTICOS}

Foi quase unânime a satisfação estética com o resultado. Quando perguntados sobre as fotos antes e depois da cirurgia, os participantes expuseram que, hoje, estão mais satisfeitos com a própria aparência e sorriso, além de declararem não ser mais necessário disfarçar ou evitar ficar de perfil. A satisfação estética proporciona impactos positivos tanto nas relações sociais quanto na qualidade de vida do indivíduo.

Alguns sujeitos apontaram insatisfação com a cirurgia pelo resultado estético, em que pese à melhora funcional. Por exemplo, o participante $n^{\circ} 119$ disse que "a parte funcional ficou bom, mas a estética, que era o grande motivo que me levou a realizar, nem parece que eu fiz", e o ${ }^{\circ} 120$ respondeu que "a parte funcional ficou ótima, mas o que me motivou a fazer a cirurgia, que era e estética, continua igual antes de fazer a cirurgia. Não melhorou em nada. Continuo frustrada". 


\section{DISCUSSÃO}

As principais razóes para o paciente procurar pela cirurgia são, além do motivo estético, tanto facial quanto dentário, o distúrbio funcional, o qual envolve a má oclusão, as desordens temporomandibulares, a dor crônica ou intermitente, a limitação dos movimentos mandibulares e o ruído na articulação, as dores na face e, algumas vezes, a dor no ouvido ${ }^{9-10}$. Como observado nos resultados, além dessas limitações presentes no paciente com deformidades dentofaciais, algumas restrições fazem parte do pós-operatório imediato, tais como o inchaço e a parestesia. Diante disso, as mudanças emocionais estão em todo o processo, sendo observada a influência que estas exercem diante do período pré e pós-operatório.

A estética facial é um fator significativo para o relacionamento entre as pessoas. Outrossim, a ideia de beleza gera um impacto na identidade pessoal e fisiológica, estando intimamente relacionada com a imagem facial e a autoestima. Por conseguinte, as deformidades dentofaciais podem influenciar a personalidade, as atitudes e o comportamento do indivíduo, de modo que a insatisfação gerada pela aparência possa desencadear problemas de ordem psicológica ${ }^{11}$.

Em decorrência disso, cabe destacar que a aparência facial é uma motivação importante para que o paciente procure o tratamento cirúrgico ortognático, já que a beleza na sociedade atual goza de extremo valor, pelas influências de diversos âmbitos mediadores da imagem corporal, como as mídias sociais, a pressão sociocultural e os padrões de beleza ${ }^{9,12,13}$. Essa teoria é corroborada pelo presente estudo, que identificou, dentre os pacientes insatisfeitos com o pós-cirúrgico, uma frustração com o resultado estético, independente de resultados funcionais satisfatórios. Ademais, foi observada a permanência de comportamentos de isolamento e a dificuldade de autoaceitação diante da nova aparência.

Um dos participantes, por exemplo, atribuiu nota "9" para a sua autoestima antes da cirurgia, e, para o pós-cirúrgico, deu nota "5", comentando o seguinte sobre o pós-operatório: "Insegura com a opinião de tudo e de todos, neurótica com o inchaço e querendo ver resultados rápidos. Aliás, estou com 7 meses e ainda me acho estranha". A imagem do corpo é considerada de grande importância para o ser humano, entre outros fatores, por estar ligada diretamente à formação da identidade das pessoas. O que o indivíduo sente e pensa a respeito do seu corpo influencia as relações sociais e é influenciado por elas, não havendo dúvidas de que o meio onde se insere interfere no desenvolvimento da autoimagem e da aparência corporal, como é o caso das deformidades dentofaciais ${ }^{14}$.

Nessa conjuntura, a qualidade de vida também é considerada subjetiva e multidimensional, a qual é capaz de compreender aspectos positivos e negativos da vida, sendo, portanto, a percepção que o indivíduo apresenta quanto à sua posição na vida, no âmbito da cultura e dos sistemas de valores segundo os quais ele vive, e em relação aos seus objetivos, expectativas, padrões e preocupações 5 .

Muitos pacientes não procuram conhecer, de forma detalhada, o procedimento, ou muitos sabem somente informações repassadas pelos outros e, por isso, não apresentam claro conhecimento acerca da cirurgia. O desconhecimento sobre o processo de recuperação e reabilitação pode desencadear conflitos emocionais no indivíduo, como depressão e arrependimento pela decisão cirúrgica tomada (como observado nos resultados). Nessa perspectiva, reforça-se a necessidade de um bom preparo e de orientações que possibilitem ao paciente vivenciar a cirurgia de forma positiva ${ }^{15}$. Dessa forma, é sugerido o acompanhamento psicológico como uma possível estratégia para manejo das emoções, tanto no período pré quanto no pósoperatório.

O paciente afetado por deformidades dentofaciais pode necessitar da cirurgia ortognática para melhora de seu bem-estar físico, psicológico e funcional, posto que o impacto ocorre em sua autoimagem, autoconceito e anatomia ${ }^{16}$. Como ponto relacionado, identificou-se que as constantes dores de cabeça ligadas à deformidade são ausentes ou pouco frequentes após a cirurgia. Melhorias funcionais significativas foram apontadas pelos sujeitos da pesquisa, os quais declararam manifestar, agora, facilidade de respiração e de mastigação, como também melhor qualidade do sono, da audição, da mordida e da fonação. Esses 
dados também revelam que o procedimento trata as deformidades dentofaciais, melhora a oclusão, adequa o posicionamento dos maxilares e realiza a harmonização facial, além de beneficiar a condição respiratória e a estética dental, ocasionando, consequentemente, maior satisfação, por exemplo, com a resolução de possíveis dores musculares e/ou articulares ${ }^{17}$.

Os corpos são afetados por inúmeros fatores sociais, tais como classe social e grupos (familiar, religioso, profissional etc.). Nesse sentido, o corpo se apresenta como espaço primordial da identidade do indivíduo, sendo influenciado pelos diferentes contextos com os quais dialoga e, simultaneamente, transformando-se em modo de afirmação pessoal ${ }^{18-19}$. A partir de estudos com jovens, foi notado que relatos sobre não pertencimento são carregados de baixa autoestima, confirmando a conjuntura social envolvida no procedimento ortognático ${ }^{20}$. Isso posto, foi verificada a permanência de déficits nas relações interpessoais dos participantes envolvidos na pesquisa, visto que a percepção que estes apresentam de si não sofreu alteração.

No entanto, padrões de comportamento e relações sociais podem manter-se na fase pósprocedimento, tendo-se em vista que se tornam inerentes à pessoa após esta ter passado muitos anos com incontáveis desconfortos. Pode-se ter uma ideia sobre esse problema na seguinte resposta sobre desconfortos sociais: "Tenho fobia social mesmo depois de operada, não sei interagir em público". A fobia social é um transtorno mental caracterizado pelo medo ou ansiedade em excesso diante de uma ou mais situações em que o indivíduo é exposto à provável avaliação de outras pessoas ${ }^{21}$. Sendo assim, pode representar um quadro de sofrimento intenso e de perdas de oportunidades para o sujeito (prejuízos na esfera profissional, pessoal ou social) ${ }^{22}$.

Quando um paciente solicita recursos odontocirúrgicos para a reestruturação da aparência, ele também busca ressignificar sua imagem pessoal e social, posto que as influências da cultura e das deformidades dentofaciais são extremamente decisivas para a estrutura de personalidade do sujeito ${ }^{23}$. Diante disso, à medida que se considera a força que o ambiente, as pressões socioculturais e os padrões estéticos exercem sobre o sujeito, é possível elhor compreensão de como o paciente com deformidade dentofacial se sente diante das relações.

A literatura menciona que dificuldades de aceitação da imagem corporal são complexas e podem ocasionar baixa autoestima, assim como mal-estar psicológico. Os pacientes com anomalias faciais também podem apresentar sintomas de transtorno de estresse póstraumático e isolamento social causado pelo estigma ${ }^{24,25}$. Esse sinal é aceito como um determinante social de saúde relevante, visto que pode gerar o sofrimento psíquico, o atraso na busca de tratamento e, até mesmo, o abandono deste e a perda da sua eficácia, o que torna essa questão uma problemática de particular interesse para a saúde pública $^{26}$.

Para efeito de comparação, vale mencionar a participação do profissional de psicologia na realização de cirurgia bariátrica, em que a avaliação psicológica é vista como uma etapa indispensável do tratamento e o paciente já é inicialmente encaminhado para o psicólogo durante o processo de preparação para o procedimento. Assim como a cirurgia ortognática, a bariátrica envolve fatores como autoestima, autoimagem e comportamento social. Isso posto, observando-se a importância já validada cientificamente da avaliação e do acompanhamento psicológico nesse contexto, é necessário atentar também nessa participação em processos cirúrgicos ortognáticos. Testes psicológicos são de uso exclusivo do profissional da psicologia, e esses instrumentos do tipo projetivo/expressivo permitem maior compreensão da personalidade do sujeito e dificilmente são manipulados ${ }^{27-28}$.

\section{CONSIDERAÇÕES FINAIS}

Perante o exposto, a cirurgia ortognática proporciona melhorias consideráveis a pacientes com deformidades dentofaciais, como nos contextos emocional e psicológico, sobretudo na autoestima. Ademais, ainda que a maioria dos participantes tenha apontado benefícios nos âmbitos mencionados, esses sujeitos também estão propícios a riscos (como em qualquer cirurgia) e a reagirem de discrepantes formas diante do procedimento. Muitos deles veem a cirurgia como algo essencial e, por isso, a depender do resultado, poderá ser gerado certo sentimento de frustração. 
Diante disso, é necessário o acompanhamento de uma equipe multidisciplinar de saúde, especialmente com a presença do psicólogo. No período pré-cirúrgico, o psicólogo deve fazer uma avaliação psicológica aprofundada, podendo optar pelo uso de testes (especialmente projetivos/expressivos) validados, para analisar de forma mais clara e assertiva os possíveis impasses sofridos pelo paciente. É válido também o uso da psicoeducação para esclarecimentos e orientações acerca de todo o processo, enfatizando-se as questões emocionais e identitárias. Nessa etapa, também é exequível trabalhar com as crenças disfuncionais do paciente quanto à cirurgia e, por conseguinte, promoverlhe maior capacidade de resiliência. No tocante ao pós-operatório, é possível ao psicólogo atuar na readaptação do paciente à sua nova face, ressignificando sua autoimagem. Ademais, o profissional pode realizar acolhimentos de possíveis agravos emocionais causados pelas mudanças ou pelas limitações que o período de recuperação acarreta.

Em referência ao presente estudo, foram observados alguns entraves durante a fase da escrita e, principalmente, na divulgação, coleta e análise dos dados do questionário na plataforma digital. No que concerne à escrita, houve limitação quanto aos referenciais teóricos do campo da psicologia que abordam o tema em questão na atualidade. Houve também baixo índice de respostas nos primeiros dias após a postagem do questionário no grupo da rede social em que foi publicada a pesquisa, demandando-se uma divulgação mais frequente por parte dos pesquisadores. Após isso, houve progresso na obtenção das respostas, no entanto, alguns dos sujeitos somente responderam ao questionário após longo período, fato este que dificultou a coleta e a análise final dos dados.

Assim sendo, existem poucos estudos acerca do tema sob a ótica da psicologia. Sendo assim, cabe ressaltar a necessidade de uma ampliação da presente investigação e a sua execução em pesquisa de campo in loco para aplicação de entrevistas e observação, permitindo-se maior vigor nos dados coletados.

\section{REFERÊNCIAS}

1. Good J, Jones R, Sood V, Kelly C. Can we justify combined orthodontic and orthognathic surgery treatment?. Orthodontic Update. 2018;11(3):100104.

2. Cariati P, Martínez R, Martínez-Lara I. Psycho-social impact of orthognathic surgery. Journal of Clinical and Experimental Dentistry. 2016;8(5):540.

3. Silva S, Teixeira V, Ferreira AP, Ustrell-Torrent MJ. Autoestima e deformidade dentofacial: um estudo comparativo com a escala de autoestima global de Rosenberg. Revista Portuguesa de Estomatologia, Medicina Dentária e Cirurgia Maxilofacial. 2016;57(3):146-150.

4. Sena RMC, Maia EMC. A utilização do constructo da autoestima nas pesquisas em saúde no Brasil: contribuições conceituais à prática clínica. Humanidades Médicas. 2017;17(2):383-395.

5. Dini GM, Quaresma MR, Ferreira LM. Adaptação cultural e validação da versão brasileira da escala de Auto Estima de Rosenberg. Rev. Soc. Bras. Cir. Plást. 2004;19(1):41-52.

6. Vale V. Do tecer ao remendar: os fios da competência socio-emocional. Exedra. 2009;(2):129-146.

7. Ambiel RA, Benetti SPC, Moreira TC. Produção científica em avaliação psicológica no contexto educacional: enfoque nas variáveis socioemocionais. Avaliação Psicológica. 2015;14(3):339-346.

8. Moraes R, Galiazzi MC. Análise textual: discursiva. Editora Unijuí. 2007.

9. Susarla SM, Tveit M, Dodson TB, Kaban LB, Hopper RA, Egbert MA. What are the defining characteristics of the most cited publications in orthognathic surgery?. International Journal of Oral and Maxillofacial Surgery. 2018;47(11):1411-1419.

10. Broers DL, Heijden GJ, Rozema FR, Jongh A. Do patients benefit from orthognathic surgery? A systematic review on the effects of elective orthognathic surgery on psychosocial functioning and patient satisfaction. European Journal of Oral Sciences. 2017;125(6):411-418.

11. Matos CCD, Rosa MEK, Figueiredo SEFMR, Barbosa 
DFM. Cirurgia ortognática e a imagem corporal. Rev. Odontol. Univ. Cid. São Paulo. 2015;27(1): $20-25$.

12. Coelho FD, Carvalho PHB, Fortes LDS, Paes ST, Ferreira MEC. Insatisfação corporal e influência da mídia em mulheres submetidas à cirurgia plástica. Rev. Bras. Cir. Plást. 2015;30(4):567-573.

13. Jensen SH. The psychosocial dimensions of oral and maxillofacial surgery: a critical review of the literature. Journal of Oral Surgery. 1978;36(6):447453.

14. Silva DR, Herzberg E. Desenho da Figura Humana: avaliação da imagem corporal na deficiência física. Aval. Psicol. 2017;16(1):106-115.

15. BarelI PS, SousaI CS, PovedaI VB, Turrinil RNT. Ansiedade e conhecimento de pacientes submetidos a cirurgia ortognática no pré-operatório. Revista Brasileira de Enfermagem. 2018;71:2206-2211.

16. Pachêco-Pereira C, Abreu LG, Dick BD, Canto GL, Paiva SM, Flores-Mir C. Patient satisfaction after orthodontic treatment combined with orthognathic surgery: A systematic review. The Angle Orthodontist. 2016;86(3):495-508.

17. Pereira RMA, Souza GMMD, Rocha VADC, Morimoto S, Tedesco TK, Mello-Moura ACV. Evaluation of the Post Orthognathic Surgery Satisfaction: a Comparative Cross-Sectional Study of Patients Class II and III. Journal of Craniofacial Surgery. 2017;28(7):1833-1836.

18. Rodrigues JC. Tabu do corpo. São Paulo: Fiocruz; 2006.

19. Carvalho SR, Guerra EM. Corpo, sociedade e construção de identidade. Ces Revista. 2015;29(2):50-66.

20. Vaz AC, André BP. O sentimento de pertencimento de alunos do bairro da rasa em armação dos Búzios/ RJ. Inter Science Place. 2016;11(4):170-194.

21. American Psychiatric Association. DSM-5: Manual diagnóstico e estatístico de transtornos mentais. Porto Alegre: Artmed; 2014.

22. Santos LF, Pires EU. Fobia social em adolescentes: repercussões acadêmicas. Revista de Psicologia da IMED. 2016;8(2):172-184.
23. Carvalho L, Melo J, Cavalcante T. Cirurgia ortognática e seus efeitos na harmonia facial: revisão de literatura. Revista da AcBO. 2019;8(1):6164.

24. Ribeiro RA, Enumo SRF. Estresse e estratégias de enfrentamento da fissura labiopalatina por préadolescentes. Saúde e Pesquisa. 2018;11(2):267276.

25. Sousa A. Psychological issues in oral and maxillofacial reconstructive surgery. British Journal of Oral and Maxillofacial Surgery. 2008;46(8):661664.

26. Atay A, Peker K, Günay Y, Ebrinç S, Karayazgan B, Uysal Ö. Assessment of health-related quality of life in Turkish patients with facial prostheses. Health and Quality of Life Outcomes. 2013;11(1):11-19.

27. Flores AC. Avaliação psicológica para cirurgia bariátrica: práticas atuais. Arq Bras Cir Dig. 2014;27(1):59-62.

28. Silva FG, Silva TCS, Nunes IFR, Costa LOC, Carneiro EB. Avaliação psicológica no pré-operatório para cirurgia bariátrica: uma revisão sistemática. Psicol. \& Conexões [periódicos na Internet]. 2019 [acesso em 1 julho 2019];1(2). Disponível em: http://revistapuca.estacio.br/index.php/ psicologiaesuasconexoes/article/viewArticle/6663.

Recebido em: 12/04/2019

Aceito em: 04/07/2019 